\title{
„NEIN, HIER BIN ICH FREMD, ICH GEHÖRE DAHIN, WO ICH NICHT BIN“. FREMDHEIT UND HEIMATSUCHE IM SCHAFFEN VON ANNA MITGUTSCH
}

In ihrem neuesten Werk Die Annäherung (2016) entwirft die österreichische Schriftstellerin Anna Mitgutsch eine weitgespannte und nachdenklich stimmende Geschichte einer durch Fremdheit und Kälte gekennzeichneten Vater-Tochter-Beziehung, sowie das Bild der schmerzvollen Rückkehr in die Vergangenheit und die Gräuel des Zweiten Weltkrieges. Im Mittelpunkt des Familienromans steht der nach einem Schlaganfall zum Pflegefall gewordene 96-jährige Protagonist Theo, der sich im Angesicht des nahenden Todes Zeit nimmt, sein bisheriges Leben Revue passieren zu lassen und alles Versäumte nachzuholen. Bevor ihn die sich einschleichende Demenz, „die Leere, die alle Wörter löscht", jeglicher Erinnerungen beraubt, ruft er aus seinem Gedächtnis verblasste Bilder der alten Zeiten auf, er denkt an seine Vorfahren aus den Karpaten, seine Kindheit und seine Kriegsjahre im Osten. Er nimmt den Leser auf seine Reise in die Vergangenheit mit, lässt ihn seine Erfolge und Niederlagen miterleben und die Fremdheit der zerrütteten Menschenverhältnisse mitempfinden. Fremd erscheint dem Protagonisten seine verstorbene Ehefrau, vor der ihn eine zu große Kluft von Missverständnissen und Schweigen trennte,

als dass er sie noch hätte erreichen können, selbst wenn er die Kraft dafür aufgebracht hätte. Jetzt, fünfzig Jahre nach ihrem Tod, erschien sie ihm wie eine Fremde in einem fremden Land, die durch ihr kurzes Leben gegangen war und auf ihrer flüchtigen Durchreise keinen Anlass gesehen hatte, sesshaft zu werden (Mitgutsch 2016, S. 11).

Fremd bleibt Theo seine eigene Tochter, die ihm schon als Kind ihre Überheblichkeit demonstrierte: „Schon als Kind war sie ihm manchmal ein wenig fremd gewesen mit ihrem Eigensinn, wenn sie ihn mit ihren hellgrauen schrägen Augen fixierte, seinen Blick festhielt und ihn zwang nachzugeben“ (ebd., S. 25). Auch

* Dr. Aneta Jurzysta, Universität Rzeszow, Philologische Fakultät, Institut für Germanistik, Abteilung für Literaturwissenschaft und Kulturwissenshaft Deutschsprachiger Länder. E-Mail: a.e.jurzysta@gmail.com 
die gealterte und bereits pensionierte Frieda weckt in dem Protagonisten keine väterlichen Gefühle: „Sie tat ihm leid, weniger weil sie sein Kind war, dessen Kummer ihn bedrückte, sondern eher wie eine Fremde, deren Elend er sah und wusste, er konnte ihr nicht helfen" (ebd., 27). Auch für die Tochter ist Theo keine liebevolle Vaterfigur, auch er scheint Geheimnisse zu hüten, die ihn als eine ihr unbekannte Person erscheinen lassen: „In solchen Augenblicken sah sie ihn an wie einen Fremden, dem sie grundsätzlich misstraute“ (ebd., S. 29).

Diese Fremdheit, die die familiären Beziehungen Theos auszeichnet, beeinflusst auch das friedliche Zusammenleben der Nationen nach dem Zweiten Weltkrieg, an den sich der Protagonist nur ungern erinnert. Einer der Gründe für die Missverständnisse zwischen ihm und der Tochter Frieda ist nämlich die Kriegsvergangenheit Theos, die auch nach Jahren im Dunkeln liegt. Während sich der Alte ins Schweigen zurückzieht und allein mit sich selbst und seinen Kriegserlebnissen hadert, macht sich seine längst erwachsene Tochter zu ihrer Lebensaufgabe, die Wahrheit über seine Kriegsjahre und die mögliche Schuld an dem Naziverbrechen aufzudecken. Die familiären Treffen werden zu regelmäßigen Verhören, auf die er mit einer Mischung aus Überdruss und Furcht wartet. Die mangelnden Informationen können der Geschichtelehrerin den Vater näher bringen, ihre Relation richtigstellen und die Fremdheit und Kälte der Beziehung aufheben. Sie will von dem alten Vater seit Jahren ein Geständnis erzwingen, „etwas, womit wir beide leben können, und dann gäbe es so etwas wie Frieden zwischen uns“" (ebd., S. 269). Seit ihrer Schulzeit ringt sie mit ihm um dieses eine Thema, was sie jahrzehntelang entzweit. Auch nach dem Schlaganfall werden die Kontakte der Familienmitglieder durch Kälte gekennzeichnet:

\footnotetext{
Wenn es nichts Bestimmtes zu tun oder zu besprechen gab, fühlten wir uns miteinander unbehaglich. An Nachmittagen, nachdem wir Kaffee getrunken hatten, trat verlegenes Schweigen ein, wir saßen einander am Küchentisch gegenüber, die Hände um die leeren Kaffeetassen gefaltet, und wie zwei Fremde, die sich gezwungen sehen, Konversation zu machen, suchten wir krampfhaft nach einem Gesprächsstoff, der nichts Schmerzliches berühren und doch persönlich sein und Nähe schaffen sollte (ebd., S. 99).
}

Die hartnäckige Spurensucherin, die die illegal in Theos Haus arbeitende Mila in ihrem ukrainischen Heimatdorf aufspüren und zu ihm zurückbringen soll, bekommt die Chance, sich nochmals an seine soldatische Vergangenheit zu heften, da ihr der Vater überraschend sein Kriegstagebuch mit auf den Weg gibt. Von diesem Bericht verspricht sich Frieda die endgültige Antwort auf die heikle Frage, ob ihr Vater, entgegen seinen lebenslangen Beteuerungen, sich als Wehrmachtsangehöriger schuldig gemacht hat und für den Massenmord mitverantwortlich bleibt. Die unternommene Reise und die unerwartete Lektüre werden zu einer Spurensuche in der Vergangenheit, zu einem Versuch der nie geglückten Auseinandersetzung zwischen der Kriegsgeneration und den Nachgeborenen. Sie soll des Weiteren der Wiederherstellung der gestörten Vater-Tochter-Beziehung dienen und die 
Fremdheit überwinden, die zwischen den Familienmitgliedern herrscht. Auch die ukrainische Pflegerin, die den Lebensabend Theos versüßt, bleibt für Frieda (aber nicht selten auch für den Protagonisten selber) eine Unbekannte, die sich in der Fremde der neuen Heimat nicht zurechtfindet und schließlich in ihr Land zurückreist. Doch die Fragen bleiben offen, die Identität des fremden Vaters verhüllt und die im Titel angekündigte Annäherung nur schwer zu erreichen: „Jetzt rächten sich die Jahre erzwungener Entfernung, es gelang uns nicht, unsere Zuneigung zu zeigen, und unsere Liebe fiel als stumme Trauer auf uns zurück“ (ebd., S. 100).

Das in dem zehnten Roman von Anna Mitgutsch dargestellte facettenreiche Motiv der Fremdheit und Kälte aller zwischenmenschlichen Beziehungen ist zu einer Konstante geworden, die ihr ganzes umfangreiches Schaffen auszeichnet. Auch die schmerzvollen Reisen in die Vergangenheit und die Erinnerungsprobleme sind in ihrem früheren Schaffen mehrfach thematisiert worden. Die Autorin beweist mit ihrer neuesten literarischen Produktion, dass sie den einst gewählten Themen treu bleibt, was nach der Darstellung der Fremdheit und der Spurensuche in der Geschichte in den früheren Werken der freischaffenden Autorin aus Linz erneut fragen lässt. Für die österreichische Schriftstellerin, die zahlreiche Reisen in den Nahen Osten, nach Südostasien oder und in die USA unternommen hatte, verschmilzt das Erfahrungs- und Erinnerungsmaterial zu einem literarisch-fiktiven Kunstwerk. Dank der eigenen Biographie mit den Vorteilen, sowie Schattenseiten der Migration vertraut, lässt Mitgutsch gesammelte Eindrücke in ihren Werken auftauchen und spiegelt sie in den Schicksalen ihrer Figuren. Die Protagonisten der bis jetzt erschienenen Romane sind beinahe ausschließlich Reisende und Migranten, sie begeben sich auf Reisen, bleiben oder kehren zurück, fliehen vor aufgezwungener Heimat und Identität und sind durch ihre Suche nach Glück, Zuhause und „wahrer“ Identität charakterisiert.

Die Schriftstellerin selber wurde am 2. Oktober 1948 in Linz geboren ${ }^{1}$, wo sie auch ihre Kindheit verbrachte und das Realgymnasium der Kreuzschwestern besuchte. Sie studierte Germanistik und Anglistik in Salzburg und schrieb ihre Dissertation über englische Lyrik der 60er Jahre. Vor dem Studienabschluss ging sie für ein Jahr nach Israel, um in einem Kibbuz zu arbeiten. Im Zeitraum von 1974 bis 1978 arbeitete sie als Assistentin am Institut für Amerikanistik an der Universität Innsbruck, zwischen 1978 und 1979 hielt sie mehrere Gastvorträge an den Universitäten von Hong Kong, Kuala Lumpur, Jakarta und Jogjakarta. In dieser Zeit blieb sie auch Gastprofessorin am Institut für Anglistik/Amerikanistik der Ewha Women's University (Seoul, Korea) tätig. Bis 1985 arbeitete sie an den Germanistikabteilungen in Bronxville, Boston und Medford. Im Jahre 1986 kehrte sie nach Europa zurück und machte mehrere Lehraufträge an den österreichischen

\footnotetext{
${ }^{1}$ Die wichtigsten Informationen zu Mitgutschs Vita kann man ihrer Internetseite entnehmen, die außerdem die Beschreibungen der publizierten Werke enthält und die wichtigsten Pressestimmen zitiert (Vgl. www.anna-mitgutsch.at, Stand: 06.02.2017).
} 
Universitäten in Salzburg und Innsbruck. Im Jahre 1996 wurde sie als Visiting Assistant Professor im Foreign Language Department an der Lafayette College in Easton, dann als Writer-in-Residence in Oberlin und Meadville engagiert. Nach der Rückkehr nach Österreich hielt sie Poetikvorlesungen sowohl an der Universität Innsbruck, als auch an der Universität Graz. Außerdem arbeitete sie im Schreibseminar am Schweizerischen Literaturinstitut Biel an der Fachhochschule Bern (2007) und hatte einen Lehrauftrag am Institut für Sprachkunst an der Universität für Angewandte Kunst Wien (2011/2012). Seit 1985 lebt sie hauptsächlich in Österreich und arbeitet als freischaffende Autorin.

Die Schriftstellerin, die mit ihrem ersten Roman Die Züchtigung (1985) breites Aufsehen erreichte und sichtbares Interesse der Leser und Kritiker weckte, betont in den Interviews, seit ihrer Kindheit davon geträumt zu haben, sich durchs Schreiben ausdrücken zu können:

Seit meiner Volksschulzeit war es mein sehnsüchtigster Berufswunsch, den ich mir jedoch nicht einmal probeweise anzumaßen wagte, Schriftstellerin zu werden. Er war von der Art von Träumen, nach denen man als Kind noch greift, die man als Jugendliche für sich behält und irgendwann als zu hoch gegriffen mit vielen anderen Erwartungen der Realität unterordnet (zit. nach Zobl 2009, S. 191).

Zu Anna Mitgutschs wichtigsten Werken gehören Das andere Gesicht (1986), Die Ausgrenzung (1989), In fremden Städten (1992), Abschied von Jerusalem (1995), Haus der Kindheit (2000), Familienfest (2003) und Zwei Leben und ein Tag (2007). In den letzten Jahren sind Wenn du wiederkommst (2010) und Die Annäherung (2016) entstanden. Seit ihrem Debüt verfasst Mitgutsch zwar zahlreiche Rezensionen, Essays, kurzprosaische Texte, Vorträge und Aufsätze, doch ihre wichtigste literarische Beschäftigung scheint immer noch das Romanschreiben zu bleiben:

Ich glaube, meine Begabung liegt im Roman. Zur erkenntnistheoretischen Bedeutung des Erzählens: Irgendwo greifen Leben und Literatur ja doch ineinander, die beiden Bereiche lassen sich nie streng trennen und ich stelle mir sowohl im Leben auch wie beim Schreiben immer die Frage, wie ist meine Erfahrung zu deuten, was bedeutet sie [...] Schreiben ist also immer ein Versuch, Erfahrungen zu interpretieren mit Hilfe von Erinnerungen, mit Hilfe von allen Erklärungsmodellen, die man eben zur Hand hat, und das ist es, was sich dann zu einem Roman verdichtet (zit. nach Höfler 2004, S. 89).

Immer wieder hebt Mitgutsch in den Interviews hervor, selbst an kein Land oder keine Kultur gebunden zu sein, sich in der Welt umschauen und in andere Identitäten kurzfristig schlüpfen zu wollen, eigene Grenzen zu erkunden und eine Weltbürgerin zu sein. So wird auch die Welt ihrer Romane von verzweifelten Nomaden bewohnt, die ihre Heimat zurückgewinnen bzw. ihr Zuhause, ihren Platz in der weiten Welt entdecken wollen. Der Prozess der Heimatsuche verlangt nach entsprechendem zeitlichem Rahmen, greift auf längst vergessene oder verdrängte Ereignisse zurück, holt die Vergangenheit hervor und erfordert 
mühsame Gedächtnisarbeit des Einzelnen. Diese ist bei Mitgutsch nicht von der Erinnerungsfähigkeit und- kultur der ganzen Völker zu trennen und mit dem kollektiven Gedächtnis verbunden, womit sich ihre Romane in die zeitgenössische Erinnerungsliteratur, sowie den seit Jahren andauernden Erinnerungsdiskurs einschreiben. In ihrem Gesamtwerk setzt sich Mitgutsch mit dem spezifisch österreichischen Gedächtnis und dessen Fähigkeit der sondierten Erinnerung und der Verdrängung der NS-Vergangenheit kritisch auseinander, womit sie nicht selten Abneigung und Kritik der eigenen Volkes erntet.

Die in ihren Romanen präsentierte Vergegenwärtigung einer bisher verschwiegenen Vergangenheit, die krampfhafte und schmerzvolle Suche nach eigenen Wurzeln, die Heimatfindung und der Heimatverlust, die mit der Suche nach der eigenen Identität einhergehen, sowie die alle zwischenmenschlichen Beziehungen determinierende Fremdheit, sind sichtbare Leitmotive ihres umfangreichen Oeuvres. Fremdheit und Heimat, Heimat und Behaustheit bilden für Mitgutsch Oppositionspaare und diese Konstellationen werden in ihren Werken in all ihren Facetten thematisiert. Das Zusammenspiel der Fremdheit und der Heimatlosigkeit wird von der Autorin so ausgelegt:

Das sind verschiedene Ebenen, die alle existieren, und zwar nebeneinander und übereinander; Ebenen, die einander verstärken und abschwächen können. Fremdsein ist für die Mehrheit der Menschen eine Grundbefindlichkeit. Heimatlosigkeit hat zwei Seiten, eine schöne und eine schreckliche: Einerseits führt Fremdsein und Entfremdung in die absolute Einsamkeit. Auf der anderen Seite ist Heimatlosigkeit eine ungeheure Chance, denn sie bringt uns Menschen auch dazu, aus der oft dumpfen Masse der Gesellschaft, die nie hinterfragt wird, herauszutreten. Ich glaube, Fremdsein ist zur Individuation des einzelnen notwendig (Teuchtmann 2003, S. 226).

Die Auseinandersetzung der Schriftstellerin mit der Fremdheit resultiert nicht zuletzt aus der ambivalenten Einstellung zu dieser Kulturerscheinung, die Julia Kristeva treffend auf den Punkt brachte: „Aber was uns an den Zügen des Fremden in Bann zieht, spricht uns an und stößt uns zurück, beides zugleich" (Kristeva 1990, S. 13). Fremdheit als interdisziplinäres kulturelles Phänomen, wie es Alois Wierlacher einmal auslegte, ist eine universelle, menschliche Erfahrung, sie impliziert „das normativ und kognitiv Fremde, die intra- und interkulturelle Fremde, die ethnische Andersartigkeit, die Außenseiter und Ausgegrenzten, das Unbekannte als das Bedrohliche oder exotisch Reizvolle und intellektuell Attraktive, das Ausländische oder Nichtzugehörige, das zeitlich oder räumlich Entfernte, das Verdrängte, Rätselhafte und Unheimliche oder die Unbegreiflichkeit Gottes...“ (Wierlacher 1993, S. 39). All die genannten Nuancen der Erscheinung werden in Mitgutschs Werken in die Figurenkonstellation und das Romangeschehen eingebaut. Man kann bei ihr also sowohl kulturelle als auch soziale Fremdheit registrieren, sie bezieht sich auf einzelne Menschen oder ganze Nationen und Generationen, sie prägt beinahe alle zwischenmenschlichen Beziehungen, die Mitgutschs Protagonisten auf ihrem Lebensweg eingehen. 
Man hat in der Mitgutsch-Forschung der letzten Jahrzehnte sowohl das Thema der Heimaterfahrung und des Heimatverlustes (u.a. Winter 2006), des immer noch nicht aufgearbeiteten Holocausts (Wimmer 2001, Mistewicz 2009) und der Erinnerungsarbeit (Neissl, 2003) erforscht, als auch die Erscheinung der (kulturellen) Fremdheit (Kecht 2000, Zsigmond 2009, Wieser 1993, Hack1 2009), die Identitätssuche (Cornejo 2003 und 2005), die Familienbände (Lawnikowska-Koper 2010) oder die Romanfiguren als Träger der nationalen Mentalität und Produkte der Zeit beschrieben, in der sie leben müssen. Dabei scheint in Mitgutschs Werk ausgerechnet das Zusammenspiel der Heimatsuche und der Fremdheit von besonderer Bedeutung zu sein, wie es allen voran in Romanen wie Abschied von Jerusalem oder Das Haus der Kindheit zu registrieren ist. In beiden Werken der österreichischen Autorin wagen die Hauptfiguren eine Rückkehr in die Vergangenheit, um ihrer jüdischen Herkunft auf die Spur zu kommen, sowie eigene Heimat und Identität zu finden. In beiden Romanen werden die Protagonisten als Fremde angesehen und müssen selbst die Fremdheit erleben und erleiden.

In Mitgutschs viertem Roman Abschied von Jerusalem (1995) kreisen die Gedanken der Autorin und des Lesers ununterbrochen um das Thema der Kulturund Religionsfremdheit und der sichtbaren Kollision von anziehender Vertrautheit und äußerster Distanz. Man wird von der Schriftstellerin kurzerhand dazu eingeladen, die Protagonistin Dvorah auf der Suche nach der eigenen Identität und den jüdischen Wurzeln zu begleiten, die auf dem israelisch/palästinensischen Boden mühsam voranschreiten wird. In dieser farbenprächtigen Hommage an die multikulturelle und multikonfessionelle Stadt wird der Rezipient nicht in das historische, sondern in das heutige Jerusalem entführt, wo infolge der Intifada und des kriegsähnlichen Zustands das friedvolle Zusammenleben von diversen ethnischen Volksgruppen sich als schwierig, ja sogar unmöglich erweist. Überzeugt in dem Werk der Österreicherin die Sorge um Details in der Beschreibung der Stadt und seiner Bewohner, die liebevolle und poetische Darstellung der unvergleichbaren Atmosphäre, des Geruchs oder Alltagsrealität, die der Autorin aus zahlreichen Aufenthalten wohl vertraut war: „Viel von der Naturatmosphäre stammt von meinen Reisen nach Israel. Das habe ich mir halt geleistet, die Landschaftsbilder in neugefundenen Metaphern, man muß ja nur die Schönheit nehmen, die vor einem liegt" (Gauß 1995a, S. 99). Weniger ausdrucksstark wirkt dabei die etwas schemenhafte Figurengestaltung, es wird dem Leser viel Aufmerksamkeit abverlangt, um die fragmentarischen, scheinbar teilnahmslos nebeneinander aufgelisteten Informationen zu einer sinnergebenden Einheit zu verbinden, die zahlreichen Erzählstränge und inhaltlichen Zusammenhänge zu begreifen: „The author creates a kaleidoscope of fragments from the different strands oft he novel, a colourful mosaic of life in Jerusalem as well as of Dvorah's and Sivan's relationship, interspersed with frequent flashbacks o the past" (Stone 1998, S. 169). Diese Rückblicke in die Vergangenheit beziehen sich auch auf die schmerzhafte 
Auseinandersetzung mit dem Holocaust und seinen jahrelangen Auswirkungen, unter denen nicht nur die persönlich am Kriegsgeschehen teilnehmenden Vertreter der älteren Generation, sondern auch ihre Nachkommen weiterhin zu leiden haben, da im Bereich der Vergangenheitsbewältigung ,noch vieles in Österreich geleistet werden muß“" (Schneider 1996, S. 173). Die Unterbrechungen in Dvorahs Jerusalem-Darstellung dienen der Möglichkeit, dem Leser zerstreute Einblicke in ihre familiäre Vorzeit und Ereignisse aus der österreichischen Kindheit zu gewähren, die sie nachhaltig geprägt haben. Bei den Großeltern in Österreich aufgewachsen und christlich erzogen verspürt die Protagonistin seit ihren ersten Jahren eine unerklärliche Angst und Verschwiegenheit der Familienmitglieder, sie leidet an der Unmöglichkeit, nach der Ursache dieses dubiosen Verhaltens fragen zu dürfen: „Meine Kindheit war voll von Unsicherheit, Geheimnissen und Tod. Das Schweigen war nicht nur die Schweigsamkeit alter Menschen, es hatte einen doppelten Boden, es klang hohl, und sein verzerrtes Echo konnte ich in Andeutungen und Blicken finden, in den Gesprächen der Verwandten, in Fotos, die ich nicht sehen haben durfte“ (Mitgutsch 1997, S. 69). Das Familiengeheimnis wird erst am Sterbebett der geliebten Großmutter ans Tageslicht gebracht, ihre versteckten, jüdischen Wurzeln erweisen sich als Grundlage der Ignoranz und der Anspielungen der Verwandtschaft und sie rufen bei der Protagonistin Schuldgefühle hervor: „Ich fühlte mich eines Vergehens schuldig, das ich nicht begangen haben konnte, an dem ich dennoch Anteil hatte, all die Jahre verfolgte mich die Angst, man könnte mich bei irgendeinem Schwindel ertappen und mich überführen“ (ebd., S. 80-81). Die Zugehörigkeit zum Judentum, die in Österreich der Nachkriegsjahre anscheinend weiterhin als Schande angesehen wird, lässt Dvorah nach ihrer Herkunft fragen und eigene Identität erneut festlegen. Die Wirklichkeit der weiblichen Hauptfigur wird auf einmal komplex (Vgl. Evans 2007, S. 93-106), die Protagonistin glaubt sich in einer Falle gefangen, aus der sie keinen Ausweg findet, balanciert zwischen zwei unterschiedlichen Welten, von denen keine als Heimat bezeichnet werden kann. Ihr Geburtsland wurde für sie nie zu einem Vaterland: „Ich habe mich in jeder Stadt und jedem Land mehr zu Hause gefühlt, als da, wo ich geboren bin“ (Mitgutsch 1997, S. 84).

Dvorah kann weder die christliche Erziehung und die Kindheit in einem westeuropäischen Land ablegen, noch die jüdische Tradition uneingeschränkt annehmen, von der sie zuerst nichts weiß und sich die hebräische Sprache und den jüdischen Lebensstil erst mühsam aneignen muss. Die Kluft zwischen dem Christentum und dem Judentum ist Quelle der inneren Zerrissenheit und des ständigen Leidens: „Ich habe noch niemandem beide Teile zugleich enthüllen oder erklären können: indem ich den einen zur Schau stelle, verleugne ich den anderen und auch seit ich mich entschieden habe, rächt sich der andere, indem er mich bezichtigt, mich aus den falschen Gründen bei den Juden aufgedrängt zu haben“" (ebd., S.117). Die Notwendigkeit, zwei sich widersprechende Identitäten vereinbaren zu müssen, führt bei der Protagonistin zur Erschaffung einer Wunschwelt, in der sie 
beide Seiten ihrer Persönlichkeit ablegen und fern der Lebenswirklichkeit leben darf. Dieser Versuch, auf diese Weise mit der Heimatlosigkeit und Identitätskrise fertigzuwerden, wird allerdings letztendlich misslingen und die weibliche Hauptfigur nicht mehr wissen, wer sie wirklich ist:

Was hat mein $\mathrm{Pa}$ mit mir zu tun, es stimmt nicht, daß er meine auf den kürzesten Nenner gebrachte Identität enthüllt, gar nichts verrät er, vor zehn Jahren hatte ich einen anderen Paß und eine andere Identität, und auch das war nicht ich, ich bin längst über diese nichtssagenden Daten hinausgewachsen, mein Paß lügt, es ist der Paß einer anderen (ebd., S. 231-232).

Die einzige Möglichkeit, die dunkle Vergangenheit zu erhellen, alle Familienrätsel zu lösen und zu einer Heimat zu gelangen, könnte für die junge Österreicherin das Treffen mit der während der Nazizeit in Palästina verschollenen Tante Martha darstellen, „dem Schlüssel zu etwas, das ich nicht begriff und nicht benennen konnte" (ebd., S. 79). Die verzweifelte Suche nach der nie gesehenen Verwandten wird für die Protagonistin zu einer Lebensaufgabe, die den Sinn ihres Lebens ausmacht und der sie sich gänzlich opfert: „Ich war ja besessen von ihr, wie ein Detektiv von seinem Opfer, sie war der Motor, der meinem Leben seine Richtung gab“ (ebd., S. 127). Als österreichische Katholikin geboren, entscheidet sich Hildegard als Erwachsene, der jüdischen Familiengeschichte und -tradition zu folgen, zum Judentum zu konvertieren und unter dem angenommenen hebräischen Namen Dvorah nach Israel zu fliegen. Das Bedürfnis, sich auf die Suche zu machen, kann auch als Fluchtmöglichkeit aus der sie umgebenden Realität ausgelegt werden, sei es während jeder Reise nach Jerusalem, sei es bei der endgültigen Rückkehr nach Österreich. Den Zusammenhang erklärt Dvorah in folgender Aussage: „Ob ich noch einige Tage hierbleibe oder nicht, ändert nichts an der Tatsache, daß ich auf der Flucht bin. Oder auf der Suche, das ist die andere Seite derselben Bewegung“" (ebd., S. 8-9).

Der erste Aufenthalt in Jerusalem dauert einige Monate, die junge Frau fühlt sich von der riesigen Stadt magisch angezogen. Die sichtbaren Unterschiede in der Lebensart und Lebensintensität faszinieren Dvorah, das Leben in Israel gleicht ihr der unbekümmerten und einzig wahren Existenz im Paradies. Auf der Suche nach Abwechslung, ihrer inneren Stimme folgend und an der Schönheit der Stadt berauscht, entscheidet sie sich also, das triste und geregelte Leben in Österreich aufzugeben und in einer Gemeinschaft mit einem französischen Musiker Gilbert in einem Kibbuz in Aschkelon zu bleiben. Die zusammen verbrachte Zeit macht sie glücklich, sie fühlt sich am richtigen Lebensort angelangt zu sein, ihr gefallen „der zeitlose Rhythmus der Tageszeiten, das An- und Abschwellen der Sonnenhitze und das gesättigte Verharren in einer Gegenwart, die keiner Zukunft entgegendrängt" (ebd., S. 39). Doch schnell stellen sich Langeweile und Überdruss auf, Dvorah sehnt sich wieder nach ihrer alten Heimat und der sie dort erwartenden Ordnung, Regelmäßigkeit und Harmonie. 
Auch die zweite Reise nach Jerusalem verstärkt die Protagonistin in dem Glauben, dort ihre wahren Wurzeln aufspüren und eben in Israel ihre geistige Heimat finden zu müssen. An der Seite ihres Mannes Alwin sammelt sie Eindrücke und Erlebnisse und versucht, sich in die Welt der Einheimischen einzuleben. Während sie sich aber den Juden zugehörig findet, ergreift ihr Ehemann für die arabischen Stadtbewohner Partei, er bemitleidet die von den Juden unterdrückten Araber und verbscheut die Repräsentanten der Peiniger: „Ich kann diese Leute nicht ausstehen, sagte er, kalt sind sie, arrogant und frech, drängen sich ständig vor, und immer mußt du ihnen auf die Finger schauen“ (ebd., S. 154). Dvorah wird von Alwin in die Abneigung immer wieder mit einbezogen und das eheliche Glück endet augenblicklich. Die liebevolle Scheinidylle platzt wie eine Seifenblase, da die beiden Eheleute sichtbar mehr unterscheidet, als richtig verbindet. Angesichts der Trennung kann die Protagonistin ihre Heimat erst richtig verorten: „Er konnte es nicht lassen zu vergleichen, hier und zu Hause, er mußte seine Heimat gegen mich verteidigen, und seine Heimat war nicht die meine“ (ebd., S. 154).

Innerlich zerrissen pendelt sie zwischen Österreich und dem Nahen Osten, der ihr immer näher steht und nach dem sie ständig Sehnsucht spürte. Ihre Beziehung zu dem Land ist viel intensiver als die eines entzückten Reisenden: „Aber wenn ich dort bin, sehne ich mich genauso wie eine unglücklich Verliebte, die demütig darauf wartet, aufgenommen zu werden, nicht mit den Trampelpfaden der Touristen abgespeist, sondern hinter die Steinmauern und Zypressen gelassen zu werden, wo sich das verborgene Leben vollzieht" (ebd., S. 36).

Nicht als schlichte Touristin will sie das Herkunftsland ihrer Vorfahren erkunden, die Unbehauste wünscht sich vielmehr in Jerusalem ihre Identität zu entdecken und ihr Zuhause zu finden. In dieser israelischen Stadt befindet sich die weibliche Hauptfigur an der Schwelle zwischen dem Touristen- und dem Einsiedlerdasein, zwischen der Identität einer Fremden und einer Einheimischen. Sie ist nicht im Stande, die Stadt zu verlassen, ohne sie besser zu ergründen: „nicht weil ich bleiben will, sondern weil ich noch nicht weg kann, weil ich gerade etwas entdeckt habe, daß mich nicht losläßt" (ebd, S. 35). Doch in der multikulturellen Stadt wird sie nicht wirklich heimisch, die Stadtbewohner sehen sie zwar nicht als eine länger bleibende Reisende, aber auch nicht als eine von ihnen an:

Die Stadt war meine größte Sehnsucht, die sich nie erfüllte, weil ich nie wußte, was ich eigentlich von ihr wollte. Jedesmal, wenn ich hier bin, schleiche ich mich um sie herum wie eine hungrige Katze, zwänge mich zwischen ihre Mauern, dränge mich auf, nicht unwillkommen, aber doch nur geduldet (ebd., S. 109).

Bei aller Bewunderung und bei allen Zugehörigkeitsgefühlen weigert sich die emanzipierte Weltbürgerin, sich der orientalischen Welt vollkommen anzupassen und sich für die hiesige Bekleidungsart zu überzeugen. Ihre westeuropäische, von heimischen Traditionen abweichende Bekleidung, sowie liberalere Verhaltens- 
weise und ziehen vor allem in arabischen Vierteln begehrliche Männerblicke an und sie stellen immer wieder Gefahr für die meist allein durch die Stadt laufende Frau dar. Auch die orientalische Denkweise muss der gebürtigen Österreicherin mit jüdischen Wurzeln zuerst erklärt werden, damit sie sich in die sie umgebende Realität besser einpassen kann. Wodurch sich Dvorah von ihren israelischen Bekannten unterscheidet, ist nämlich weniger ihr Äußeres oder ihr verschwindender Akzent, sondern ihr durchaus westliches Denken, ihr ständiges Beharren auf Logik, Gerechtigkeit und Berechenbarkeit: „Sie versucht, mir mein westliches Denken abzugewöhnen, und ich glaube manchmal, ich bin auf dem besten Weg: Es gibt nicht bloß eine Wahrheit, behauptet sie, es gibt viele und alle zugleich“" (ebd., S. 50).

So wie sich die Stadt Jerusalem zwischen dem Osten und dem Westen, zwischen verschiedenen Nationen und Religionen befindet, schwebt auch Dvorah zwischen mehreren Identitäten und Sprachen, kann keine von ihnen ablegen, nimmt westeuropäische Verhaltensweisen in die bunte israelische Welt mit. Immer wieder wird die Österreicherin gewarnt, die Gefahren der multiethnischen Riesenstadt zu umgehen, nicht zu provozieren und sich vor den Attentätern und sie belästigenden Männern zu schützen. Doch die Fast-Israeli maßt sich das Recht an, frei durch die Stadt zu wandern: „Die Einheimischen wissen es besser, ich bin ein bunter Vogel, der sich in den Netzen der Altstadt verheddert hat. Aber ich kann wählen, wohin ich gehe, sie nicht. Sie gehören ins jüdische, ins armenische oder ins arabische Viertel, ich bin überall“ (ebd., S. 179). Der Suchenden fällt es anfangs nicht leicht, kulturelle Besonderheiten und Unterschiede aufzudecken und einzuordnen, den orientalischen Geist zu begreifen, der ihr folgendermaßen beschrieben wird: „Orientalische Spiritualität, sagte Nurit, hat die verschlüsselte Poesie eines Märchens, eines verführerischen Traums, aus dem man nicht erwachen möchte. Versuch erst gar nicht, ihn zu verstehen" (ebd., S. 185). Für die Menschen, die zu Ansässigen geworden sind, ist das Leben in Jerusalem zwar ein erwünschtes Abenteuer, eine unvergleichbare Erfahrung, aber solch ein Schwelgen in einer Märchenlandschaft birgt auch viele Risiken in sich: „Dort und hier, sagte ich, das sind verschiedene Welten, auch uns ist alles hier fremd, wir leben hier wie in einem arabischen Märchen, und nichts, was wir erleben, ist ganz wirklich, wir sind den Orient nicht gewöhnt. Das kann gefährlich sein“ (ebd., S. 184-185). Die Unkenntnis der herrschenden Wertordnung und der unterschiedlichen Verhaltensnormen, die das Zusammenleben aller ethnischen Gruppen regeln, stürzt Dvorah in tiefe Unsicherheit, rastlos und chaotisch durchstreift sie endlich die fremden Straßen Jerusalems: "Ich habe mich beherrschen müssen, nicht zu rennen, wie eine Verbrecherin auf der Flucht. An der Straßenecke habe ich mich noch rasch umgedreht, als müsste ich mich orientieren, doch niemand schien mir zu folgen" (ebd., S. 7).

Das Gefühl, beobachtet und verfolgt zu werden, stellt sich nach dem Ende einer sehr riskanten Beziehung mit Sivan an. Die Bekanntschaft mit dem in einer 
Synagoge kennengelernten Mann, der sich als christlicher Armenier und Dolmetscher vorstellt, dessen Identität aber bis zum Ende der Beziehung im Dunkeln bleibt, lässt Dvorah alle Warnungen vergessen und sich auf eine Liebschaft mit dem Unbekannten einlassen. Der Schönling Sivan versinnbildlicht nämlich die verbotene Frucht, das Gefährliche und Fremde. Er ist das „Bedrohliche“, „Dunkle“, aber auch andererseits das „Erregende“ und „Anziehende“(Vgl. Gauß 1995b). Die Protagonistin ist sich dessen bewusst, dass der gutaussehende Sivan sie möglicherweise nur ausnutzt, weil sie eine Europäerin ist, oder sich durch eine Liaison mit einer Jüdin an den verhassten Zionisten rächen will. In der Zweisamkeit haben Zweifel aber keinen Zugang zu der verliebten Frau, sie scheint vollkommen realitätsblind zu sein. Sonst aufrichtig und zuvorkommend, ändert Sivan in dem palästinensischen Viertel sein Verhalten, er wirkt auf einmal distanziert, aggressiv und fremd. Seine Hassausbrüche gegenüber den Israelis, die er für die Schikanen, Demütigungen, Armut und Aussichtslosigkeit der Palästinenser verantwortlich macht, zeigen sein anderes Gesicht und lassen in der Protagonistin Schuldgefühle aufkeimen: „Wir waren Feinde, er beschuldigte mich stellvertretend, und je mehr er mich beschuldigte, um so weniger war ich zur Einsicht, die er mir abverlangte, bereit“ (Mitgutsch 1997, S. 227). Dvorah fühlt ihre Zugehörigkeit zum jüdischen Volk, alle Schmerzen, die es jemals ertragen musste, aber auch die Verantwortung für jegliche Fehler und Vergehen der jüdischen Nation. Die Meinungsverschiedenheit, die nicht zuletzt auf unterschiedlicher Herkunft beruht, führt erneut (wie vor Jahren in der Ehe mit Alwin) zum Ende der Beziehung Dvorahs. Beide Parteien verheimlichen vor dem Partner ihre wahre Identität und ihre Wurzeln, beide weigern sich, im Namen der Liebe die Maske abzunehmen und alle Geheimnisse preiszugeben. Das Gefühl kann es nicht vollbringen, feindliche Lager zu versöhnen: „Wir waren Feinde, wußten es zum erstenmal voll hilfloser Trauer. Es war eine Feinschaft ohne Haß, in der die Liebe sich in verletztes Schweigen hüllte“ (ebd., S. 260).

Die Liebschaft mit dem einheimischen Unbekannten endet abrupt, Sivan verschwindet und sein Bild wird in einem Bericht über eine Schießerei abgedruckt, laut dem Sawad Radadeh sich unter den vier getöteten Terroristen befand. Bis zum Schluss bleibt seine wahre Identität ein Rätsel, die Neugier auf den geliebten Unbekannten wird immer größer, er selber aber „mit jeder Erinnerung fremder“ (ebd., S. 216). Erst nach dem Verschwinden des anfänglichen Charmeurs ist Dvorah im Stande, die Identität des Liebhabers zu bezweifeln und auch nach der eigenen erneut zu fragen. Statt ihre eigene in Jerusalem aufzubauen und sich selbst zu finden, lief sie nämlich an seiner Seite die Gefahr, sich vollkommen zu verlieren. Die Stadt, die zum Schauplatz gefährlicher amourös-politischer Spannungen wurde, ihr ihre Wurzeln zeigen und ihre Vergangenheit zurückgeben sollte, beraubt sie stattdessen beinahe der Gegenwart und der Zukunft. Es bewahrheitet sich, was man ihr einmal von der Wirkung der Metropole verraten hat: ,Jerusalem macht jeden meschugge“" (ebd., S. 169). 
Tief betroffen und einsam sieht Dvorah schlussendlich ein, dass Sivan sie zu seinen eigenen Zwecken und zur Vorbereitung eines Terroraktes benutzt hat (um leichter Grenzkontrollen zu passieren) und sie selber das Volk ihrer Vorfahren in Gefahr bringen konnte. Wie einst, als sie von ihren jüdischen Wurzeln erfahren hat, überfallen die Protagonistin Schuldgefühle: „Ich bin aus der Unschuld gefallen, jetzt erst, obwohl ich schon längst schuldig bin. Jede Bombe, die einen überfüllten Bus zerreißt, könnte mit meiner Hilfegelegt sein, jeder durch Terror Ermordete könnte auf meine Rechnung gehen“ (ebd., S. 174-175).

Die Rastlosigkeit, mit der die Protagonistin durch Jerusalems Viertel läuft, ist als Symbol für ihre innere Hoffnungslosigkeit und ihr Verlorensein aufzufassen. Die Verfolgungswahn nimmt unaufhaltsam zu, Dvorah befürchtet, von allen Mitmenschen bespitzelt zu werden. Jeder Einheimische, mit dem sie sich seelen- und blutsverwandt fühlte, stellt jetzt eine Gefahr für sie dar, kann ein Verräter sein, der ihr unheimlich ist: ,wie ich den Augenblick fürchte, in dem er aus dem Schatten tritt und sich zu erkennen gibt" (ebd., S. 244). Einst bewunderte Stadtbewohner werden der Protagonistin fremd, auch sie selbst kann sich in dem labyrinthischen Stadtdschungel nicht mehr zurechtfinden: „Nichts kam mir bekannt vor (...). Feindseligkeit lag in der Luft, man nahm mich wahr und sah durch mich hindurch, die Dunkelheit verdichtete sich zu stockfinsterer Nacht" (ebd., S. 280).

Der Entschluss Dvorahs, Jerusalem wieder zu verlassen, resultiert aus der Verzweiflung über den Verrat Sivans und aus dem endgültigen Bodenverlust. Die Jüdin soll somit nach Österreich und in ihre alte Identitätskrise zurückkehren, aus der sie keinen Ausweg gefunden hat, der Kreis schließt sich somit ab. Alte Probleme werden mit der Rückkehr wieder lebendig, alte Fragen scheinen erneut aktuell zu sein, ob sie immer noch Hildegard blieb, wie sie ursprünglich hieß, bevor sie den neuen Vornamen annahm, oder schon Dvorah geworden ist. Sie scheint letzten Endes keine eindeutig festgelegte Identität zu besitzen, sie fand sich in der vergangenheitsverhafteten österreichischen eingesperrt, entwarf sich laut ihren Wurzeln eine jüdische, konnte sich aber mit keiner der beiden vollkommen identifizieren. Die Reise in das Land der Vorfahren bat der Protagonistin keine Heilungskur für die Persönlichkeit an, sie ist sich auch dessen bewusst, dass die Rückkehr in die ihr fremde Welt schmerzvoll sein wird, da man dort für ihre Nöte kein Verständnis aufbringt: ,Wenn ich nach Hause komme, werden sie mir wieder den seit meiner Kindheit gehassten Namen aufnötigen, mich hineinzwängen in ein Ich, das sie sich für mich ausgedacht haben, und glauben, sie täten mir ein Gefallen und nähmen mich so, wie ich bin“ (ebd., S. 77). Der Hauptfigur des Romans bleibt somit nur die Fluchtmöglichkeit, die ihr früher geholfen hat, sie aber zu einer Fremden inmitten der Familie und der Gesellschaft machte: „Immer schon, seit meiner Kindheit, habe ich mir in Verkleidungen gefallen und mir ein anderes Leben erfunden, um mir und dem Zufall... zu entfliehen“ (ebd.).

Der jüdischen Vergangenheit muss sich auch der Hauptprotagonist des umstrittenen Romans Das Haus der Kindheit (2000) stellen, in dem Anna Mitgutsch 
erneut Themen anschneidet, die als Erkennungszeichen ihres Schaffens fungieren können. Zur Sprache bringt die Autorin also die Suche nach der Heimat, die Wurzellosigkeit und die Fremdheit des Einzelnen in der ihn umgebenden Wirklichkeit, sowie auch die österreichische Vergangenheitsbewältigung, die Prozesse der Erinnerungsverdrängung, die Enteignung der Juden im Dritten Reich oder langwierige Rückstellungsverfahren nach 1945. Hat der Leser im früheren Werk in Jerusalems orientalische Atmosphäre eintauchen dürfen, so wird er durch Mitgutschs ,großen Roman" (Hackl 2000, S. 84) über die Enteignung und Sehnsucht nach Behaustheit (Vgl. Mitgutsch 1999, S. 156) „beinahe trunken von den ungemein dichten Orts- und Naturbeschreibungen, den Schilderungen des Alltags in New York und in amerikanischen Kleinstädten“" (Hackl 2000, S. 84).

Im Unterschied zu ihren vorher erschienenen Werken, in denen überwiegend Frauen als Erzählerinnen und Hauptdarstellerinnen zu Wort kamen, gilt das Interesse der Schriftstellerin und des Lesers diesmal einer männlichen Figur, die in Österreich geboren und in den USA aufgewachsen jetzt in die österreichische Kleinstadt H. zurückkehrt, um das bereits im Titel angekündigte „Haus der Kindheit“ zurückzuerobern. An die frühen Jahre in Österreich kann sich der Emigrant Max Berman nur sehr bruchstückhaft erinnern, wenn schon, dann kann er in seinem Gedächtnis nur idealisierte Landschaftsbilder oder Gerüche aufrufen:

Der glitzernde Fluß, der die Stadt in großem Bogen teilte, und der farblose Himmel verschmolzen zu einem durchsichtigen Leuchten, und dieses Bild blieb Max im Gedächtnis haften als das Lebensgefühl seiner frühen Kindheit, nach dem er sein ganzes Leben strebte: Helligkeit, Weite, die festtägliche Stille eines nie zu Ende gehenden Sommernachmittags (Mitgutsch 2000, S. 12).

Das Aussehen der Menschen aus seiner europäischen Kindheit hat der damals Fünfjährige längst vergessen, in seiner Vorstellung tragen die Erwachsenen genau die Gesichter, „die ihnen die wenigen Fotos aus jener Zeit gegeben hatten. Sie trugen sie wie Masken“ (ebd., S. 10) (Vgl. Teuchtmann 2002, S. 50-53).

Die Lebensjahre in Österreich, die Max mit Geborgenheit und Glücksgefühlen assoziiert, enden abrupt mit der Notwendigkeit, das Land zu verlassen und nach Amerika zu emigrieren. Während dem Vater Saul der amerikanische Lebensstil und die neuen Lebensverhältnisse gut gefallen und er die gewonnene Heimat sichtlich genießt, kann sich die verzweifelte Mutter Mira in dem amerikanischen Traum nicht zurechtfinden und der neuen Umgebung nicht anpassen. Den Aufenthalt in Amerika versteht sie als eine Übergangslösung, eine kurze Episode, nach der sie ihre alte Existenz wieder aufnehmen darf: „Sie war zeitlebens Europäerin geblieben, klassenbewußt, der Vergangenheit und einem gepflegten, mitunter dünkelhaften Lebensstil zugeordnet" (Mitgutsch 2000, S. 16). Die immer lebenslustige und zuversichtliche Mira verliert auf dem fremden Boden zunehmend ihre Vitalität und ihren Optimismus. Genau diese Angst und Traurigkeit der Mutter 
bringt der Hauptheld in Erinnerung, wenn er an die Zeit unmittelbar nach der Ankunft zurückdenkt. Unterschiedliche Lebenseinstellungen, finanzielle Probleme und sich wiederholende Streitereien münden unweigerlich in die Trennung der Eltern und haben den Umzug der Familie nach Brooklyn zur Folge, womit sowohl der soziale Abstieg der Bermanns, als auch die Annäherung der Familienmitglieder an die jüdischen Traditionen anfängt.

Es ist die enge Bindung des Protagonisten an die Mutter, die sein Bedürfnis nährt, nach Österreich zurückzukehren, es ist das alte schwarz-weiße Foto des Hauses, das ihn (wie die Mutter Mira) an die Vergangenheit fesselt. Das alte Bild, das die verlorene Vergangenheit, Glückszeit und Sicherheit symbolisiert, wird als einzige Erinnerung an die Heimat als größter Schatz gehütet, pietätvoll in jeder neuen Wohnung aufgestellt und wie eine "Schwur" verstanden, die einen dazu verpflichtete, ,ein Versprechen einzulösen“ (ebd., S. 7)2. Nicht das reale Haus in H., sondern die kleine und scheinbar bedeutungslose Fotografie erweckt bei dem Protagonisten die Erinnerung an die geliebte Mutter und den unüberwindbaren Wunsch, auch ihr zu Ehren in das verlassene Haus einmal zurückzukehren. Sie gilt als ,sicherstes Indiz einer Vergangenheit, die nicht mehr existiert, als fortexistierender Abdruck eines vergangenen Augenblicks“" (Gürtler 2004, S. 74).

Die Beziehung zwischen den beiden seelenverwandten Familienmitgliedern bleibt bis zum Lebensende Miras sehr innig, der Mutter Wünsche und Phantasien werden zu den ihres Sohnes:

Die Nähe zwischen ihnen bedurfte keiner Worte. Sie war in der schwärmerischen Verehrung für die in seinen Augen konkurrenzlos schöne Mutter verankert, in ihren gemeinsamen Träumen und Geschichten aus einer Vergangenheit, in der es nur glückliche Tage gegeben hatte, und in dem lebhaften Wunsch des Sohnes, ihr dieses Glück zurückzugeben (Mitgutsch 2000, S. 26).

Die Vergangenheit auszulöschen und das Haus ihrer Jugend zurückzuerobern, wird zu einer verhüllten Lebensaufgabe ihres jüngsten Sohnes (Vgl. Fliedl 2013, S. 295). Die ihm von der Mutter vererbte Sehnsucht nach der alten Heimat führt Max zuerst in den 40er Jahren in der Uniform eines amerikanischen Soldaten in seine Herkunftsgegend, doch das alte Haus hat bereits neue Besitzer, die mit dem Amerikaner nicht einmal reden wollen. Die in Österreich verbliebenen Familienmitglieder sind inzwischen ermordet worden, was Mira zu Tode betrübt und jeglicher Illusionen beraubt. Ab sofort weigert sie sich, Deutsch zu sprechen, und die alten Zeiten in Österreich zu erwähnen: „Sie ließ sich durch keine Erinnerungen mehr trösten- alles, was einmal Hoffnung und Trost gespendet hätte, verwandelte sich in Schmerz" (Mitgutsch 2000, S. 36).

Im Laufe der Jahrzehnte baut sich der Protagonist sein persönliches und vor allem berufliches Leben auf: Das jüdische Einwandererkind verwirklicht den

\footnotetext{
${ }^{2}$ Die Bedeutung des Bildes als eines Erinnerungskatalysators beschreibt Höfler (2008).
} 
amerikanischen Traum und wird zum geschätzten Restaurator, der sich mit Luxus umgibt und sich nichts verbieten muss. Nur das alte Haus des Großvaters bleibt ihm unzugänglich, das in seinem Geburtsland sich im fremden Besitz befindet. Im Laufe der Jahre wacht in dem Protagonisten, der sich eigentlich in das neue soziale Umfeld integriert hat, das Leben in der Metropole sichtlich genießt und sich bisher selber als einen richtigen Amerikaner verstand, die Sehnsucht nach der Geborgenheit des Familienhauses, nach der Zurückeroberung der alten Heimat auf. Es war vielleicht seiner Mutter Sehnsucht, die sich in seiner Phantasie eingenistet hat, die sich jahrelang „,betäuben ließ, aber die nie verstummte“ (ebd., S. 46) (Vgl. Rosenfeld 2000, S. 638). Der unerfüllte Wunsch Miras, in die Heimat zurückzukehren, der Verlust der Nächsten sowie die sie umgebende Fremdheit bewirken, dass sie sich immer deutlicher abkapselt, von den Wahnvorstellungen heimgesucht wird und endlich einem Schlaganfall erliegen muss. Ihr Sohn entscheidet sich erst 1974 in die Stadt H. zu reisen, um das langwierige Rückstellungsverfahren für die arisierten Güter der Familie einzuleiten. Mit seinem Vorhaben und seinem Auftreten löst er ausschließlich spürbar negative oder zumindest sehr distanzierte Reaktionen bei der nichtjüdischen Bevölkerung der Kleinstadt aus (Vgl. dazu Bushell 2004, S. 85-103). Auch er misstraut den Bewohnern des österreichischen Ortes: „Einerseits wissen sie von nichts, andererseits behandeln sie einen mit dieser Mischung aus Unterwürfigkeit und Überheblichkeit. Und wenn man sie anspricht, schauen sie beleidigt weg“" (Mitgutsch 2000, S. 81). Wie in Abschied von Jerusalem wird auch in diesem Roman das Problem unterstrichen, dass die österreichische Bevölkerung sich der Wahrheit über den Zweiten Weltkrieg und die Judenermordung nicht stellen wollte und seine Anteilnahme an dem Verbrechen gerne verschwieg. Das Verhüllen und Verleugnen der nationalsozialistischen Vergangenheit kann der Emigrant nicht nachvollziehen, das mangelnde Schuldbewusstsein, das beharrliche Schweigen der Einheimischen, die ihre ehemaligen jüdischen Nachbarn scheinbar aus dem Gedächtnis ausgelöscht haben, vertiefen zusätzlich die Kluft und intensivieren die Fremdheitsgefühle zwischen dem Amerikaner und den Österreichern: „Es war ein fremdes Haus in einer fremden Stadt, die ihm wie ein Lebewesen vorkam, das sich stur fortpflanzte und die Vergangenheit überwucherte“"(ebd., S. 110).

Wie Dvorah in Jerusalem meint auch Max in seiner Geburtsstadt ständig beobachtet und verfolgt zu sein. Mehr als jemals zuvor und stärker als in anderen Städten, in denen er sich vorübergehend aufgehalten hatte, fühlt sich Max in H. unentwegt darauf verwiesen, daß er fremd war: „Es waren die flinken, aufmerksamen Blicke, die es ihm bestätigten, und jedesmal bei seinem ersten Satz spürte er, wie alle aufhorchten und sich fragten, woher er komme" (ebd., S. 94). Nur mit den wenigen zurückgekehrten jüdischen Stadtbewohnern kann Max also kommunizieren, die den Amerikaner in ihren Kreis miteinbeziehen wollen. Mit einer Jüdin Nadja geht Max Berman eine Liebesbeziehung ein, er ermöglicht ihr auch die Ausreise nach New Jersey, nachdem er Österreich wieder hat verlassen müssen, da er das 
Haus bis zum Tode der aktuellen Besitzer nicht alleine verwalten durfte. Zeit seines Lebens ist er aber außer Stande, langanhaltende Beziehungen aufzubauen und aufrechtzuerhalten, die Enge des Verhältnisses scheint ihm die persönliche Freiheit zu rauben, was unweigerlich zu Reibereien und schließlich zu der endgültigen Trennung führen muss. Max leidet unter Bindungsängsten, seine Liebe bleibt der jungen Frau verwehrt und beide Partner zum ewigen Alleinsein verurteilt.

Nur der berufliche Erfolg und der Gedanke an die Zukunft im zurückgewonnenen Haus des Großvaters geben seinem Leben einen Sinn. Nach achtzehn Jahren erreicht ihn endlich die erfreuliche Nachricht, dass er der alleinige Besitzer der österreichischen Familiengüter ist und dem Einzug in das Haus somit nichts mehr im Wege steht. Sein innigster Wunsch scheint damit in Erfüllung zu gehen:

Jetzt war die Zeit gekommen, und mit dem Alter war die Sehnsucht nach den Kindheitserinnerungen gewachsen, ihren Gerüchten, Stimmen, den Schatten und dem Licht in den Räumen zu verschiedenen Tageszeiten, die er wiedererkennen würde wie zurückkehrende Bilder aus einem dem Vergessen entrissenen Traum (ebd., S. 166).

Gesundheitlich angeschlagen wird es sich des Alterns und der schwindenden Kräfte bewusst, der Großstädter will sofort die verlorenen Jahre zurückholen, alles für den Lebensabend Aufgehobene zeitnah erleben. Doch der jetzige Zustand hält den verklärten Erinnerungen aus der Kindheit und dem Schönheitsideal aus dem Bild nicht mehr stand. Das zu einem palastähnlichen Anwesen stilisierte Gebäude gleicht in der Wirklichkeit einem engen Arbeiterhaus. Auch die kostenaufwendigen Renovierungsarbeiten vermögen es nicht, den negativen Eindruck zu löschen, die alte Wirklichkeit wiederzubeleben: „Max ging durch die Zimmer. Keines entsprach den Vorstellungen, die er sich all die Jahre gemacht hatte. Klein waren sie, und er hatte sie immer groß in Erinnerung gehabt (...) Das Haus war fremd und abweisend, es enttäuschte ihn" (ebd., S. 179).

Zwischen den Jahren der Emigration und der langersehnten Rückkehr sind über sechzig Jahre verstrichen, die nicht nur die Umgebung und die Erinnerungen an die alte Heimat veränderten, aber auch eine Evolution der Persönlichkeit und der Bedürfnisse des Protagonisten herbeiführten. „Aber das Haus seiner Kindheit ließ sich nicht zurückgewinnen. Je weiter die Restaurierungsarbeiten voranschritten, desto mehr verblassten die alten Bilder. Erinnerungen ermüdeten ihn, gleichzeitig waren sie alles, was ihm an diesen einsamen Abenden geblieben war" (ebd., S. 238-239). Das renovierte Haus ähnelt nicht mehr dem Abbild auf der schwarz-weißen Fotografie der Mutter, schafft nicht wirklich das Zuhause, das er in Europa zu finden sich ausmalte. Die Erinnerungen ${ }^{3}$, die das einzige waren, was einem nach der Auswanderung und dem Heimatverlust nicht verlorengehen konnte, haben allmählich die Vergangenheit glorifiziert und die schlechten Eindrücke

${ }^{3}$ Die Macht und Bedeutung der Erinnerung in Mitgutschs Werken untersuchen u.a. Shafi (2003, S. 33-51) oder Teuchtmann (2002, S. 43-61). 
aus dem Gedächtnis getilgt (Vgl. Teuchtmann 2003, S. 58). Erst in Anbetracht der Rückkehr wird das Paradoxe an Max' Gefühlen entlarvt: Das Bild des Hauses entpuppt sich als wichtiger als das reale Haus in Österreich selbst, die im Bild und in der Erinnerung festgehaltene und somit konservierte Vorstellung stellt für ihn mehr Wert dar, als der eigentliche Gegenstand der jahrzehntelangen Sehnsucht.

Es verlangt von Max viel Mühe, sich in Österreich abzufinden und seine alte/ neue Wahlheimat zu akzeptieren, zumal er grundsätzlich mit den jüdischen Bewohnern in Kontakt steht, die nichtjüdischen dagegen weiterhin distanziert bleiben und ihn als einen Fremden ansehen, der in der Vergangenheit des Ortes wühlt (vgl. Schneider 2004, S. 50-51). Mit der zurückweichenden Lebenskraft und dem Nachlassen des Gedächtnisses verändert sich auch die Sicht des Protagonisten auf das eigene, sich dem Ende neigende Leben. Er blickt keiner Zukunft mehr entgegen, was ihn erwartet, sind lediglich das Altsein und der Tod. Melancholisch und vereinsamt verabscheut er die österreichische Idylle und die Enge des Hauses, seht sich wiederum danach, nach Amerika zurückkehren und seine Farbenfröhlichkeit und Lebenstotalität nochmals erleben zu dürfen. In der Provinz des Geburtsortes wird dem Protagonisten ersichtlich, dass er „dort, zwischen Alphabet City und Crotona Park, hingehörte“ (Mitgutsch 2000, S. 82). Um leben zu können, müsste er sich vor der Stadt und dem Haus seiner Kindheit retten, die ihn betrüben und mit der jahrhundertelangen Geschichte des Volkes belasten.

Als seine krampfhaften Versuche misslingen, Nadja zurückzuerobern und sie endlich bei einem Unfall ums Leben kommt, nachdem sie von Max damit beauftragt worden ist, die jüdischen Friedhöfe in Przemysl zu fotografieren, bricht die Welt des Protagnisten endgültig zusammen. Er bleibt mit dem überwältigenden Gefühl zurück, als Mensch vollkommen versagt zu haben. Der Tod der geliebten Menschen entlarvt die traurige Tatsache, dass der Amerikaner in seinem „Haus der Kindheit" kein richtiges Zuhause fand, dass er sich aber weder in den USA noch in Europa heimisch fühlt und dass er im Grunde kein Amerikaner und kein Österreicher mehr ist. Er bleibt in der Wirklichkeit in der Schwebe, an der Grenze zwischen zwei sich nicht ergänzenden Welten, die aber nicht als Heimat angesehen werden darf ${ }^{4}$. Wie einst seine ausgewanderte Mutter, die sich bis zum Lebensende weder in den USA noch in Europa mehr verorten konnte und unter schmerzvoller Identitätskrise zu leiden hatte, kann auch ihr Sohn seine wahre Zugehörigkeit nicht richtig einschätzen. Dem zwischen Behaustheit und Unbehaustheit, zwischen unterschiedlichen Welten, Kulturen, Kontinenten und Sprachen wandernden Restaurator fehlt anscheinend das Gefühl, irgendwo verwurzelt sein zu müssen, da seine einzige Bindung an Österreich durch die Erinnerungen seiner Mutter geprägt ist (vgl. Rosenfeld 2004, S. 34-35). Seine zwischenmenschlichen Beziehungen blieben bis auf wenige Ausnahmen distanziert und kühl, den amerikanischen Freunden erklärt Max seine Kontakte mit den Bewohnern auf folgende

\footnotetext{
${ }^{4}$ Das Bild der Grenze im Schaffen A. Mitgutschs untersucht u.a. Günther (1998, S. 179-188).
} 
Weise: „Eigentlich lebe ich in der Vergangenheit dieser Stadt, an ihrer Oberfläche habe ich mich nur vorläufig eingerichtet" (Mitgutsch 2000, S. 240). Dieses Bekenntnis stellt den Beweis dafür dar, dass Max H. und Österreich nicht als Heimat anerkennt, sonst würde er sich in die ihn umgebende Gesellschaft einfügen wollen, wie er das einst in Amerika getan hat.

Der Weltbürger aus New York beteuert in der Öffentlichkeit, dass er keine familiären Wurzeln braucht, um sich als selbständiges Individuum zu behaupten, er überzeugt vor allem sich selbst, dass man keine Vorgeschichte besitzen bzw. verstehen muss, um sein Leben in den Griff zu bekommen: „Sind Wurzeln so wichtig (...) Sind wir denn Pflanzen, daß wir Wurzeln brauchen? Ich habe (...) auch keine Wurzeln (...) es ist aufregend, ein wurzelloser Kosmopolit zu sein. Zu Hause ist man dort, wo man liebt" (ebd., S. 211). Und doch verleitet ihn der innere Suchzwang dazu, die jüdische Vergangenheit zu ergründen, eine Chronik über die Juden der österreichischen Stadt H. aufzuschreiben, in der er grundsätzlich die verschwiegenen und marginalisierten Kriegsjahre vor dem Vergessen bewahren kann. Die Ergebnisse seiner Recherchen und das traurige Bewusstsein, dass sich das Los des jüdischen Volkes ständig wiederhole, dass ihre Lebensgeschichte ein unendliches Zusammenspiel der Verfolgung und Ermordung darstelle, lässt den Protagonisten an den Sinn seiner Anstrengung zweifeln. Wie alle Juden ist er den Bewohnern fremd geworden und weigert sich endlich, unter diesen Menschen zu weilen, ,denen er so gleichgültig und unverständlich war, wie ihnen alles Jüdische durch die Jahrhunderte gleichgültig und unverständlich gewesen war“ (ebd., S. 278). Das Versprechen wurde nach Jahren erfüllt, das Haus ist zurück in den Familienhänden und die unausgesprochene Sehnsucht immer schwächer, was auch die verblasste Hausfotografie symbolisiert. Die Suche nach der Zugehörigkeit und Heimat, die gleichzeitig als Suche nach seiner eigenen Identität aufzufassen war, kann somit beendet werden: „Nichts hält ihn mehr an diesen Ort, an dem alles in die Vergangenheit weist, die Vernichtung des jüdischen Lebens, die sich nicht wegmachen läßt“" (Hackl 2000, S. 85).

Max Berman schafft es endlich, sich von dem „Haus der Kindheit“ und von den Erinnerungen zu verabschieden. Er vermag es damit auch teilweise, die Vergangenheit als ein Teil seiner Familiengeschichte und Identität hinter sich zu lassen. Die misslungene Heimkehr führt weder bei ihm noch bei der Protagonistin des Romans Abschied von Jerusalem die erwünschte Harmonie und Sicherheit herbei, die erforschten jüdischen Wurzeln liefern keine solide Existenzbasis. Die Vergangenheit bleibt durch die familiär-nationale Erinnerung oder komplizierte Herkunft in die Gegenwart der beiden Figuren integriert. Ihr treuer Begleiter bleibt bis zum Ende die Fremdheit, die sie nie loswerden können. Dabei scheint es schier unmöglich festzustellen, ob sie als folgerichtige Konsequenz des Heimatsverlustes und der Heimatunbestimmtheit zu sehen ist, oder umgekehrt das Fehlen der einzigen und endgültigen Heimat erst die Helden zu Fremdlingen und Außenseitern macht. In beiden Ländern, Österreich und Israel oder Österreich und 
Amerika, bleiben sie genauso heimatlos, in beiden Staaten plagt sie das Bewusstsein, nirgendwo richtig dazuzugehören. Die Existenz der Figuren Mitgutschs wird von Geheimnis, Verschweigen und Erinnerung bestimmt, die sie in die weite Welt treiben, ohne Antworten auf die gestellten Fragen parat zu halten.

Der Kampf um eigene Identität und die krampfhafte Heimatsuche enden bei Dvorah und Max mit der Resignation, Ausgrenzung und Einsamkeit. Weitere Versuche werden nicht mehr unternommen. Was Dvorah/Hildegard ausspricht, gilt auch für Max Berman: „Es ist sinnlos geworden, weiterhin auf den Fragen, die offengeblieben sind, zu beharren. Es gibt auch niemanden mehr, der zweifelsfrei die Wahrheit kennt (...) Ich bin nicht klüger geworden, nur ein bißchen ärmer und nüchterner“" (Mitgutsch 2000, S. 277).

\section{LITERATURVERZEICHNIS}

Bushell A., The return of the native, or the neighbours are back: Anna Mitgutsch's novel „Haus der Kindheit", In: Foster I., Wigmore J. (Hrsg.), Neighbours and Strangers. Literary and cultural relations in Germany, Austria and Central Europe since 1989, Amsterdam 2004, S. 85-103.

Cornejo R., Die Ich-Spaltung als das „Eine“ und das „Andere“ am Beispiel des Romans „Das andere Gesicht" von Anna Mitgutsch, In: „Brünner Beiträge zur Germanistik und Nordistik 10 “, 2005, S. 163-180.

Cornejo R., Fremd nicht nur „In fremden Städten “; ein Exkurs in die Fremde auf dem Weg zu sich selbst. Anna Mitgutschs Romane der 90er Jahre, In: Broser P., Pfeiferová D. (Hrsg), Der Dichter als Kosmopolit. Zum Kosmopolitismus in der neuesten österreichischen Literatur. Beiträge der tschechisch-österreichischen Konferenz České Budějovice, März 2002, Wien 2003, S. 163-175.

Evans K., Anna Mitgutsch - ,Abschied von Jerusalem. Ein Sich-Einlassen auf die Komplexität der Wirklichkeit“", In: Bushell A., Kostalova D. (Hrsg.), Von außen betrachtet. Österreich und die österreichische Literatur im Spiegel der Auslandsrezeption, Bern 2007, S. 93-106.

Fliedl K., Gebäude und Gedächtnis. Zu Anna Mitgutschs ,,Haus der Kindheit“ (2000), In: Kastberger K. (Hrsg.), Grundbücher der österreichischen Literatur seit 1945, Wien 2013, S. 292-301.

Gauß K.-M. (a), Abschied von Jerusalem. Eros und Untergang, „Literatur und Kritik“ 1995. H. 295/96.

Gauß K.-M. (b), Das Dunkle ist die Haut, In: „Die Presse“, 25.3.1995.

Günther P., Der unbewohnbarste Ort: Über den Begriff der Grenze bei Anna Mitgutsch, In: Fiddler A. (Hrsg.), ,, Other “ Austrians: post-1945 Austrian women's writing. Proceedings of the conference held at Nottingham from 18-20 April 1996, Bern 1998, S. 179-188.

Gürtler C., Abschied von einem fremden Haus, In: Die Rampe. Sonderband zu Anna Mitgutsch, Linz 2004.

Hackl E., Ahnung von einem Zuhause. Anna Mitgutschs großer Roman, In: „Literatur und Kritik“, 2000 H. 343/344, S. 84-86.

Hackl W., Fremde und Aussenseiter in den Romanen von Anna Mitgutsch, In: Bartsch K., Höfler G.A. (Hrsg.), Anna Mitgutsch, Graz /Wien 2009, S. 57-72.

Höfler G.A., Das Photo als Erinnerungskatalysator im Familienroman bei Anna Mitgutsch, Arno Geiger und Andrea Grill, In: Byczkiewicz A. u.a. (Hrsg.), Verbalisierung und Visualisierung der Erinnerung Literatur und Medien in Österreich, Łódź 2008, S. 237-252.

Höfler G.A., „Ideologie interessiert mich nicht: Aus einem Gespräch mit Anna Mitgutsch (Jänner 2001)“, In: Die Rampe. Sonderband zu Anna Mitgutsch, Linz 2004, S. 89-91. 
Kecht M.-R., Bilder der inneren Wahrheit: Fremdsein und Heimatsuche. W. Anna Mitgutsch, „Das andere Gesicht" (1986), In: Chapple G. (Hrgs.), Towards the millennium. interpreting the Austrian novel 1971-1996; zur Interpretation des österreichischen Romans 1971-1996, Tübingen 2000, S. 147-168.

Kristeva J., Fremde sind wir uns selbst, Frankfurt am Main 1990.

Ławnikowska-Koper J., Das Familienbild in Anna Mitgutschs Roman „Familienfest": Zu Strategien der Fixierung von Wandel und Auflösungserscheinungen im Hinblick auf sozialhistorische Prozesse, In: „Colloquia Germanica Stetinensia“ 18 (562), 2010, S. 285-300.

Mistewicz M., O stosunku Austriaków do swej przeszlosci na podstawie „, Abschied von Jerusalem “ $i$,Haus der Kindheit“ Anny Mitgutsch, In: „Studia Niemcoznawacze / Studien zur Deutschkunde“, 43/2009, S. 229-241.

Mitgutsch A., Abschied von Jerusalem, Reinbeck bei Hamburg 1997.

Mitgutsch A., Das Haus der Kindheit, München 2000.

Mitgutsch A., Die Annäherung, München 2016.

Mitgutsch A., Erinnern und Erfinden, Graz 1999.

Neissl J., ,, Manchmal ist das Thema mit einem Roman noch lange nicht erschöpft “. Autobiographische Erfahrungen und kollektive Erinnerungsspuren in Texten von Anna Mitgutsch, In: Kernmeyer H. u.a. (Hrgs.), Schreibweisen, Poetologien. Die Postmoderne in der österreichischen Literatur von Frauen, Wien 2003, S. 379-396.

Rosenfeld S., Anna Mitgutsch: Schreiben als Protest und Affirmation, In: Die Rampe: Porträt Anna Mitgutsch. Linz 2004, S. 33-38.

Rosenfeld S., Das Haus der Kindheit, In: „World Literature today“, 2000, Vol. 74, Nr. 3.

Schneider G. K., Abschied von Jerusalem, In: „Modern Austrian Literatur“, 29/1996, Nr. 1.

Schneider G., Die Schwelle ist mein Ort: Fremde als Schwellenpersonen in den Romanen von Anna Mitgutsch, In: Die Rampe: Porträt Anna Mitgutsch, Linz 2004, S. 45-52.

Shafi M., ,Enteignung “ und „Behaustheit“: Zu Anna Mitgutschs Roman „Haus der Kindheit“, In: „Modern Austrian Literature“, Vol. 36, No. 1/2 (2003), S. 33-51.

Stone M., Anna Mitgutsch's Abschied von Jerusalem: An Austrian Writer's Presentation of a Divided City, In: Fiddler A. (Hrsg.), „Other “ Austrians. Post - 1945. Austrian's womens writing. Proceedings oft he conference held at the University of Nottingham form 18-20 April 1996, Bern 1988, S. 167-178.

Teuchtmann K., Über die Faszination des Unsagbaren: Anna Mitgutsch. Eine Monografie, Frankfurt am Main 2003.

Teuchtmann K., Teuchtmann K., Zur Darstellbarkeit der Zeit: Erinnerung und Erfindung in Anna Mitgutschs „Die Züchtigung “ und „Haus der Kindheit“, In: „Modern Austrian Literature“, Vol. 35, No. 1/2 (2002), S. 43-61.

Wierlacher A., Fremdheit - ein Kulturthema. In: Wierlacher A., Kulturthema Fremdheit. Leitbegriffe und Problemfelder kulturwissenschaftlicher Fremdheitsforschung, München 1993.

Wieser M., Waltraud Anna Mitgutsch: Phänomenologie des Fremdseins. In: „Die Zeit und die Schrift", Szeged 1993, S. 289-296.

Wimmer A., Conditional Reconciliation: Austria 's Dark Historical Chapters in New Novels by Frederic Morton and Anna Mitgutsch. In: Steiner D. (Hrgs), American studies and peace. Proceedings of the 25th AAAS conference (5.-7.11. 1999, Salzburg, Schloss Leopoldskron), Frankfurt a.M. 2001, S. 305-314.

Winter H.-G., Zum Diskurs über Heimaterfahrung und -verlust in literarischen Texten der deutschsprachigen Gegenwartsliteratur: Waltraud Mitgutsch. In: Razbojnikova-Frateva M., Winter H.-G. (Hrgs.), Interkulturalität und Nationalkultur in der deutschsprachigen Literatur, Dresden 2006, S. 351-372.

www.anna-mitgutsch.at (Stand: 06.02.2017). 
Zobl S., „Für jene, die ohne Stimme sind. Anna Mitgutsch- Ein Leben zwischen den Kontinenten“. In: Bartsch K., Höfler G.A. (Hrsg.): Dossier 28: Anna Mitgutsch. Die Buchreihe über österreichische Autoren, Band 28, Graz 2009, S. 187-195.

Zsigmond A., Fremdheitsmuster in Anna Mitgutschs Roman In fremden Städten, In: Rácz G., Szabó L.V. (Hrgs.), Der deutschsprachige Roman aus interkultureller Sicht (Studia Germanica Universitatis Vesprimiensis Supplement 10), Veszprém 2009, S. 341-356.

Aneta Jurzysta

\title{
“NO, I'M A STRANGER HERE, I BELONG SOMEWHERE ELSE!” ALIENATION IN THE LITERARY WORK OF ANNA MITGUTSCH
}

\author{
(Summary)
}

The paper deals with the broad concept of strangeness in the literary work of Anna Mitgutsch, a contemporary Austrian writer. The topic of strangeness is a keynote in her rich literary output. Mitgutsch's experience of being abroad (travelling to the Middle East and south-east Asia, living and working in Israel, England, Korea and The USA) is reflected in such novels as Das andere Gesicht, In fremden Städten or Abschied von Jerusalem. Her characters are nomads, people looking for identity and homeland, trying to escape alienation. The feeling of being a stranger is also present in their relations with their loved ones (Die Annäherung).

Keywords: strangeness, German literature, homeland, alienation 